SESSION 1. THE BASIC DATA

"Symbiotic stars are like platypus" David Allen 


\title{
A PERSPECTIVE ON THE SYMBIOTIC STARS
}

\author{
David Allen \\ Anglo-Australian Observatory \\ PO Box 296, Epping \\ NSW 2121 \\ Australia
}

ABSTRACT. I give a very brief summary of the state of our knowledge of the symbiotic stars, together with some of my hopes for how the field will develop.

\section{PREAMBLE}

The task of the introductory speaker at a conference is a challenging one. The more so since the words one might choose for the verbal presentation differ from what the reader will seek in the final publication. So, I have decided to cheat: the text you are now considering reading is not what you would have heard if you attended the conference ... well, only partly so. I have taken the liberty of using different titles for the two presentations, to reflect their distinct emphases. But in one important way I have not cheated. This paper was written before the conference, and has not subsequently been modified. It may contain errors that are corrected by later papers; that is the risk I take. On the other hand, it is as fair a summary as I can give, as useful an introduction as I can conceive, to the view of symbiotic stars prevalent early in August 1987. I sincerely hope that the papers which follow will so overthrow the contents of this introduction that you will have no interest in reading it twice.

What I will present in the limited space available here cannot be regarded as a review, but only as a perspective. I eschew references (subsequent papers surely contain ample) save to draw attention to the proceedings of the 1981 conferences on the subject (Stencel 1981; Friedjung \& Viotti 1982), and the only book published to date on these stars (Kenyon 1986).

Although not a review, it is appropriate to illustrate this paper with one optical spectrum of a classical symbiotic star, to show just what it is that characterises these objects. Because it is in the optical that they were first recognised, and still are classified, I have not broadened the waveband. Features to note in the spectrum, reproduced on the next page, include the $\mathrm{TiO}$ bands of the cool giant in the red; the Balmer jump in emission that shows the blue continuum to be gaseous rather than stellar; the high-excitation emission lines ( $\lambda 4686$ of He II; $\lambda 6087$ of [Fe VII]); and the unidentified bands at $6830,7088 \AA$, which are markedly broader than atomic emission lines of comparable intensity. 


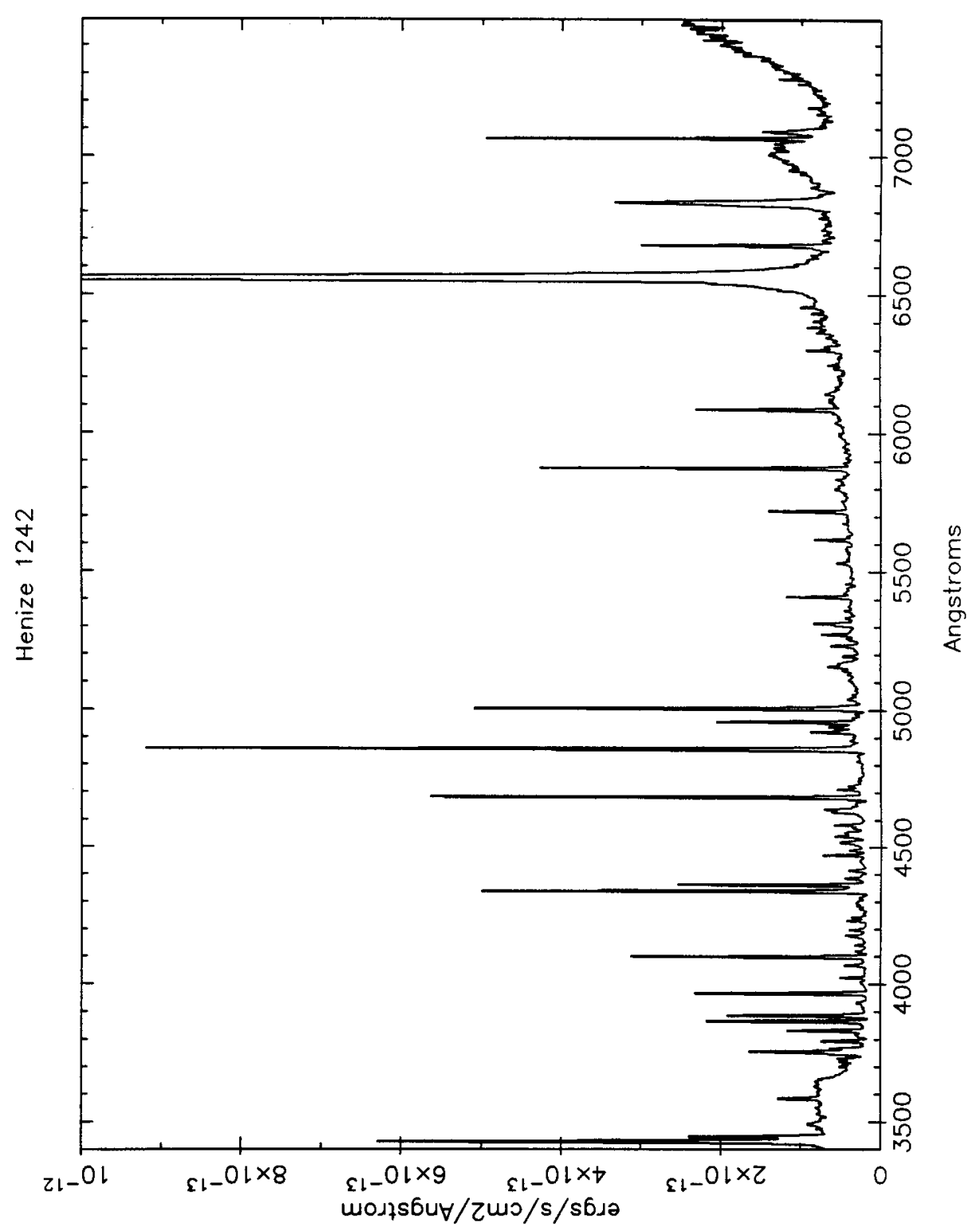




\section{SYMBIOTICS AS BINARIES}

When first found, in the 1920's, the symbiotic stars were mere curiosities: the platypuses of the stellar zoo. While a few astronomers pondered and committed their thoughts to paper, most ignored the peculiar objects. The principal characteristics of the symbiotic stars - the simultaneous display in an apparently single object of two temperature regimes differing by a factor of 30 or more - then was unique. Today, having a broader base in the electromagnetic spectrum, we take such matters for granted: witness radio galaxies, the X-ray binaries, or the Vega dust cloud.

It was just as apparent half a century back as now that the $3000 \mathrm{~K}$ component is a stellar photosphere. But we remain ignorant of the luminosity class of that star in most cases. The origin of the $100,000 \mathrm{~K}$ radiation, however, exercises but so often eludes us.

In the 1980's interacting binaries have become fashionable to describe the idiosyncracies of a range of stellar systems that previously seemed utterly bizarre. The symbiotic stars fall readily into the binary bin, and I doubt whether more than $1 \%$ of attendees at this conference will argue wholeheartedly for a single-star interpretation of the majority of symbiotic stars. I hope that some do, because it is essential not to blinker ourselves too much. But there is an undeniable elegance in the binary interpretations, and I will offend few if I posit as an introduction to this conference that symbiotic stars are interacting binary systems.

\section{SUBCLASSES OF SYMBIOTIC STARS}

I should qualify that statement. More than most taxons, that of the symbiotic stars has become the dustbin of stellar eccentrics. It is a peculiarity of our species that we need to categorise things. Consequently, we put into the symbiotic bin objects about which we know depressingly little, simply because they show a subset of the characteristics of the well-studied specimens. Many probably belong, but some equally probably do not. And some of the misfits may indeed be single objects. I therefore propose the following: of the objects currently classified as symbiotic stars, only those which are interacting binaries belong in the class.

Of course, lack of adequate data on the majority forces us to maintain a lengthy list of probable members of the class, pending a better description of each. The intention of this somewhat brutal definition is not to reduce the class to a handful of examples, but to encourage an alternative classification for any that are shown to be single objects. We may, as a result of this conference, identify a few examples that quite clearly are not binaries, or are binaries in which interaction appears irrelevant to the appearance; if we do, then let us classify them differently. My aim is to remove from the symbiotic classification some of the litter of stars that has tarnished it in the past. My personal belief is that we will lose very few from the present catalogue by this tighter definition, and that we will gain unity in our research.

Even within the binaries, however, we must recognise that there are several types of interaction that can stimulate the apparition we call a symbiotic star. At the start of the decade these various possibilities were hotly debated. We tended at that time to seek a single mechanism to account for all members of the class. In the intervening years our views have matured. It is important when approaching this subject to recognise that we are working on several distinct types of interaction, and to realise that not all papers necessarily refer to all the subclasses. Nor is it certain that we have yet identified all possible subclasses. We are nearing the stage 
at which we should agree on definitions for some of the subclasses, perhaps naming them after their best-studied type stars.

The physical conditions that appear able to produce objects classifiable as symbiotic stars are as follows:

- A main sequence star accretes by Roche-lobe overflow from a late-type giant. The source of ultraviolet luminosity is purely gravitational, and originates in an accretion disk. Variability is caused by changes in the mass transfer rate, as in cataclysmic binaries. Example (and ideal type star): CI Cyg.

- A white dwarf accretes directly from the wind of a late-type giant or mira variable. Much of the hydrogen is burnt as it accretes, providing the ultraviolet flux. The accretion rate undergoes relatively small changes, so the variability is not extreme. Example: RW Hya.

- A white dwarf, that has accreted from the wind of a late-type giant or mira variable and so accumulated unburnt hydrogen, undergoes a shell flash. An ultra-slow nova results. Example: AG Peg. The flash appears capable of persisting for many decades, raising the possibility that some underwent their slow nova eruptions before observations began. A probable example of the latter is $\mathrm{H} 1-36$.

- A neutron star accretes from the wind of a late-type giant. The only known example is V2116 Oph, the optical counterpart of GX1+4.

Even amongst these groups the question of the luminosity class remains open. Is the mass donor making its first ascent of the giant branch, or is it on the asymptotic branch? Are supergiants ever involved? And what of the small number of systems (such as $\mathrm{BD}-21^{\circ} 3873$ ) which seem to involve a $\mathrm{G}$ star?

It will be apparent that there is a danger at this stage of opening up the symbiotic class too widely. The second of the configurations listed above includes Mira (o Ceti) which has a distant white dwarf companion accreting from its wind. There are probably valuable details to be learnt from a study of Mira, but it would confuse our field to call Mira a symbiotic star. Consequently, I favour retaining an earlier definition that symbiotic stars exhibit emission of $\lambda 4686 \mathrm{He}$ II or perhaps of some equivalently ionized species. In effect, this is an untidy attempt to define a minimum temperature and luminosity for the hot companion.

The different types of symbiotic stars I listed have grossly different evolutionary states. It is possible, even, that some binaries pass through two symbiotic phases, as each member star in turn becomes the mass donor. Are the numbers of the various types compatible with evolutionary expectations? We can only answer this important question if we can classify the 130 or so known symbiotic stars into their appropriate interaction configurations. Unfortunately it has only been possible to classify a very few examples, and then by time-consuming individual study. Are there quicker ways to classify them?

It has been sugested, for example, that the velocity widths of the unidentified emission bands at $6830,7088 \AA\left(1000 \mathrm{~km} \mathrm{~s}^{-1}\right.$ is typical) are too large to be produced in the wind from or the disk around a main sequence star, and thus imply the presence of a white dwarf? Is this a valid way to recognise the white dwarf accretors? Again, the presence of forbidden lines in the spectrum indicates that considerable amounts of gas at relatively low densities are illuminated by the hot star. The absence of forbidden lines implies that all the illuminated gas is of high density, a 
circumstance that is unlikely to arise if a wind operates from either star. Can we therefore argue that systems without forbidden lines have Roche-lobe overflow? If we make both these plausible assumptions then why do we see the $6830,7088 \AA$ bands in many symbiotic stars which are devoid of forbidden lines, for Roche lobe overflow from a cool giant would surely exceed the accretion capabilities of a white dwarf.

\section{MORE QUESTIONS}

I have just raised a few of the questions for which I hope answers will soon be forthcoming. Other questions come to mind, and I list a few now. Some require further observational study, others need theoretical treatment. In some cases the present body of observational data may provide an answer if we can think it through with sufficient clarity.

- To what extent is the cool component influenced by its energetic friend? Can our classification of it be seriously confused?

- Do magnetic fields, especially in white dwarfs, contribute to the symbiotic phenomenon as they do in cataclysmic variables?

- What can we learn from the $6830 / 7088$ bands, seen in no other types of object? A challenge remains here for the atomic physicists. I stuck out my neck 8 years ago with the suggestion that they are permitted transitions in highly-ionized iron, and to my surprise nobody has challenged that view.

- How important is the wind from the accreting star? What are its effects on the accretion process? How many of the emission lines can be accounted for by a standing shock where the winds from both stars balance? Can the complex emission-line profiles in AG Peg, RX Pup and others be explained by wind interactions?

- In how many cases does an accretion disk around a white dwarf contribute appreciably to the visible and ultraviolet flux? Do we really understand accretion from a stellar wind, in particular with regard to disk formation? Since the dimensions of any such disk have a steep dependence on the wind speed of the cool star, can we improve on estimates of that parameter, for example using $\mathrm{SiO}$ masers?

- What clues have we neglected from other interacting binary systems?

- Can we find better methods than those already attempted to deduce the luminosity classes of the cool components, and hence the distances of more symbiotic stars?

- The two symbiotic stars that lie closest to us, R Aqr and CH Cyg, include jet-like radio structures. Dare we extrapolate to infer similar behaviour in other symbiotic stars? 


\section{AND SOME THOUGHTS ON WORK TO BE DONE}

Judging by the lengthy collection of papers that follows, and by their varied titles, symbiotic stars have become fashionable again. More research on them is being performed than ever before, and by more people. I suspect that symbiotic stars have become recognised as one of the topical challenges to follow the major strides that have been made in recent years in the field of cataclysmic variables. Indeed, the two recurrent novae $\mathrm{T} \mathrm{CrB}$ and $\mathrm{RS} O \mathrm{Oph}$ are claimed as both symbiotics and cataclysmics. Symbiotic stars are, undoubtedly, much more difficult subjects than cataclysmics, if only because of their grossly longer orbital periods. The recent outburst of RS Oph attracted much interest among researchers who had not previously studied symbiotic stars, and many beautiful data emerged. It is still unclear, however, whether the outburst was a shell flash on a white dwarf or an accretion event onto a mainsequence star.

Although the number of symbiotic stars with known periods is increasing, we still desperately need more. That work requires long, patient monitoring of light curves and radial velocity variations. We should not ignore other possibilities, including the use of microwave masers associated with the cool stars, and searches for periodic changes of optical polarization. There is a wealth of work for those with access to large amounts of time on small telescopes.

I see a great need for better modelling of the emission nebulae, with regard to both line strengths and velocity structure. The full treatment of a shock between the stars needs to be incorporated into these models, and with several types of symbiotic system to model there is work for a number of energetic souls. We still await treatment of the zeroth order approximation wherein an isotropic mass-loss nebula is illuminated by an external source of ionizing radiation.

The question of distances and luminosities of both components remains thorny. There is scope for some deep surveys in regions of known distance. Baade's window is one such location, and the Magellanic Clouds another. To date only carbon symbiotic stars have been located in the Clouds, but I can report the recent discovery by Morgan, Good and myself of an oxygen-rich symbiotic star in the LMC which, from luminosity arguments, must involve an asymptotic branch $\mathrm{K} 5$ giant.

\section{A CATALOGUE OF BIZARRE QUALITIES}

I have concentrated somewhat on optical matters, and it is time to redress the balance. I do so by listing some of the unexpected, or unexplained features that have shown up in other wavebands, and make no apology that a few of them have already been noted above. Scott Kenyon, in the next paper, will elaborate much more on the multifrequency nature of these stars, and subsequent papers will highlight different aspects.

- V2116 Oph is a hard X-ray pulsar and the only hard X-ray symbiotic.

- Slow X-ray flares occur during the outbursts of some symbiotic stars; otherwise only a few symbiotics are stable $\mathrm{X}$-ray sources.

- The ultraviolet continua often show more components than can readily be modelled.

- The ultraviolet emission lines of AG Peg and RX Pup are broad and complex, and differ from ion to ion. 
- R Aqr is enveloped in a vast bipolar nebula.

- The thermal emission at radio frequencies has a spectral index $(\sim 0.9)$ that is not readily explained.

- Systems containing Mira variables show greatly reduced maser emission in $\mathrm{OH}$, $\mathrm{H}_{2} \mathrm{O}$ and $\mathrm{SiO}$ than normal Miras.

- Nonthermal radio flares have been seen in at least three stars.

- Radio jets are seen in CH Cyg and R Aqr; the R Aqr jet has an optical counterpart.

\section{INTRODUCTION}

I end with an introduction, not to this paper but to this volume. Symbiotic stars are challenging objects, rewarding to pit oneself against. More than almost any other stellar types their radiation spans the electromagnetic spectrum, from low-frequency radio to hard $\mathrm{X}$-ray. There is something in them for almost every style of astronomy and astronomer. The attendance at this conference alone testifies to the burgeoning interest they are generating.

This panchromatic characteristic of the symbiotic stars does, however, mean that we must guard against blinkering ourselves. The history of the subject is sprinkled with examples where research on one facet of the stars has been described and discussed with inadequate reference to available data at other wavebands; on more than one such occasion the existing literature violated the conclusions reached.

With the publication of this volume we shall have taken another valuable step forward in understanding the symbiotic stars. Covered within its pages are virtually all facets of the subject. It should be compulsory reading for all who work on these fascinating systems.

\section{REFERENCES}

Friedjung, M. \& Viotti, R. (eds.), 1982. "The Nature of Symbiotic Stars", Int. Astr. Union Coll. 70 (Reidel, Dordrecht).

Kenyon, S. J., 1986. "Symbiotic stars" (Cambridge University Press).

Stencel, R. E. (ed), 1981. "Proc. N. Amer. Workshop on Symbiotic Stars" (Joint Institute for Laboratory Astrophysics, National Bureau of Standards and University of Colorado). 